PHYSICAL REVIEW D 95, 079901(E) (2017)

\title{
Erratum: Self-interacting dark matter and cosmology of a light scalar mediator \\ [Phys. Rev. D 93, 015016 (2016)]
}

\author{
Kimmo Kainulainen, Kimmo Tuominen, and Ville Vaskonen \\ (Received 21 March 2017; published 4 April 2017)
}

DOI: 10.1103/PhysRevD.95.079901

The true exclusion constraint from the invisible Z-decay width is less constraining than the one shown in Fig. 4 of Ref. [1]. We present a corrected version of the calculations and the figure below [2]. The invisible Z-boson decay width is

$$
\Gamma_{Z \rightarrow \text { inv }}=\Gamma_{Z \rightarrow \nu_{e} \nu_{e}}+\Gamma_{Z \rightarrow \nu_{\tau} \nu_{\tau}}+\Gamma_{Z \rightarrow \nu_{\mu} \nu_{\mu}}+\Gamma_{Z \rightarrow N N}
$$

Let us denote the decay width of $Z$ to one relativistic fermion by $\Gamma_{Z \rightarrow \nu \nu}$. If, for example, $N$ mixes only with a $\tau$ neutrino, then

$$
\begin{aligned}
& \Gamma_{Z \rightarrow \nu_{e} \nu_{e}}=\Gamma_{Z \rightarrow \nu_{\mu} \nu_{\mu}}=\Gamma_{Z \rightarrow \nu \nu}, \\
& \Gamma_{Z \rightarrow \nu_{\tau} \nu_{\tau}}=\cos ^{2} \theta \Gamma_{Z \rightarrow \nu \nu}, \\
& \Gamma_{Z \rightarrow N N}=\sin ^{2} \theta \Gamma_{Z \rightarrow \nu \nu}\left(1-\frac{m_{N}^{2}}{m_{Z}^{2}}\right)\left(1-\frac{4 m_{N}^{2}}{m_{Z}^{2}}\right)^{\frac{1}{2}} .
\end{aligned}
$$

Now for $m_{N} \ll m_{Z}$, the invisible decay width of $Z$ is $\Gamma_{Z \rightarrow \text { inv }}=3 \Gamma_{Z \rightarrow \nu \nu}$, as in the standard model. This decay width is in agreement with the experimental value given by Eq. (4.5) of the original paper. The mixing modifies $\Gamma_{Z \rightarrow \text { inv }}$ only at $m_{N} \gtrsim m_{Z} / 2$, as shown in Fig. 1 .

In addition, our CMB constraint on sterile neutrinos with $m_{N} \lesssim 1 \mathrm{MeV}$ was too weak; we only required that such neutrinos decay before CMB formation. But this is not enough: Since $N$ 's decay only to neutrinos, their late decay could lead to a significant increase in the neutrino energy density, potentially in conflict with the existing bounds on the amount of dark radiation [3]. We therefore require that $N$ energy density does not exceed that of one-half an equivalent neutrino degree of freedom at the time of their decay. Assuming that the freeze-out of sterile neutrinos occurred before the QCD phase transition $\left(T_{\mathrm{fo}}>T_{\mathrm{QCD}}\right)$, we find that at $T<m_{e}$

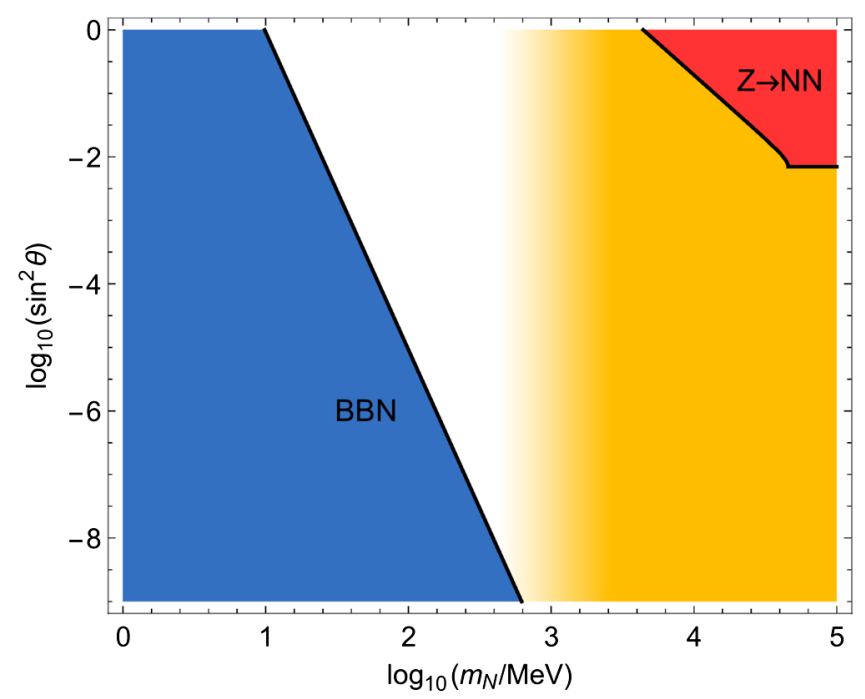

FIG. 1. We show the excluded regions in the sterile $N$ parameters due to the $Z$-decay width (red area), the BBN (blue area), and the CMB (green area). In the rightmost shaded region (yellow area), $S$ is too heavy to provide a sufficiently strong self-interaction for $\psi$. 


$$
\rho_{N}=m_{N} n_{N}=m_{N} \frac{g_{\text {eff }}(T)}{g_{\text {eff }}\left(T_{\text {fo }}\right)} \frac{3 \zeta(3)}{2 \pi^{2}} T^{3} \approx 0.008 m_{N} T^{3},
$$

which, relative to the relativistic neutrinos, is

$$
\frac{\rho_{N}}{\rho_{\nu}}=\frac{0.008 m_{N} T^{3}}{\frac{7 \pi^{2}}{120}\left(\frac{4}{11}\right)^{4 / 3} T^{4}} \approx 0.05 \frac{m_{N}}{T} .
$$

We then must require that $N$ decays at the latest at $T=m_{N} / 10$. The $N$ decay width relative to the Hubble expansion rate is given by [using Eq. (4.8) of the original paper for decay width $\Gamma_{N \rightarrow 3 \nu}$ ]

$$
\left.\frac{\Gamma_{N \rightarrow 3 \nu}}{H}\right|_{10 T=m_{N}} \approx 0.03\left(\frac{m_{N}}{\mathrm{MeV}}\right)^{3} \sin ^{2} \theta,
$$

which gives

$$
m_{N}(\sin \theta)^{2 / 3}>3 \mathrm{MeV}
$$

This excludes the gray region in Fig. 4 of the original paper. Hence, the ultimate lower bound for the mass of $N$ at given $\sin ^{2} \theta$ comes from the requirement that $N$ decays before BBN. Figure 1 shows a corrected version of Fig. 4 of the original paper.

[1] K. Kainulainen, K. Tuominen, and V. Vaskonen, Phys. Rev. D 93, 015016 (2016).

[2] We thank J. M. Cline for bringing this error to our attention.

[3] G. Rossi, C. Yeche, N. Palanque-Delabrouille, and J. Lesgourgues, Phys. Rev. D 92, 063505 (2015). 